\title{
THE STRUCTURE OF QUASINORMAL OPERATORS AND THE DOUBLE COMMUTANT PROPERTY
}

\author{
BY \\ JOHN B. CONWAY ${ }^{1}$ AND PEI YUAN WU ${ }^{2}$
}

\begin{abstract}
In this paper a characterization of those quasinormal operators having the double commutant property is obtained. That is, a necessary and sufficient condition is given that a quasinormal operator $T$ satisfy the equation $\{T\}^{\prime \prime}=\mathscr{Q}(T)$, the weakly closed algebra generated by $T$ and 1 . In particular, it is shown that every pure quasinormal operator has the double commutant property.

In addition two new representation theorems for certain quasinormal operators are established. The first of these represents a pure quasinormal operator $T$ as multiplication by $z$ on a subspace of an $L^{2}$ space whenever there is a vector $f$ such that $\left\{|T|^{k} T^{j} f: k, j \geqslant 0\right\}$ has dense linear span. The second representation theorem applies to those pure quasinormal operators $T$ such that $T^{*} T$ is invertible. The second of these representation theorems will be used to determine which quasinormal operators have the double commutant property.
\end{abstract}

1. Introduction and preliminaries. In this paper all Hilbert spaces are separable and over the complex scalars $\mathbf{C}$. Recall that an operator $T$ on a Hilbert space $H$ is quasinormal if the two parts of its polar decomposition commute. Equivalently, $T$ is quasinormal iff $T$ and $T^{*} T$ commute. Quasinormal operators were first studied by Brown [1], who derived a representation for them. (See Theorem 2.1 below.)

For an arbitrary operator $T,\{T\}^{\prime}$ is the commutant of $T$ and $\{T\}^{\prime \prime} .=\left\{\{T\}^{\prime}\right\}^{\prime}$. $Q(T)$ denotes the closure of $\{p(T): p$ is a polynomial $\}$ in the WOT (weak operator topology); equivalently, the closure can be taken in the SOT (strong operator topology) [8]. Denote by (dc) the set of operators having the double commutant property; that is, $T \in(\mathrm{dc})$ iff $\mathcal{Q}(T)=\{T\}^{\prime \prime}$. The class (dc) has received some attention. In particular, see $[6,9,12,22-25]$.

Part of the reason for the interest in (dc) is the fact that every hermitian operator belongs to $(\mathrm{dc})$. However, not every normal operator belongs to $(\mathrm{dc})$.

\subsection{Proposition. If $T$ is normal, $T \in(d c)$ iff $T$ is reductive.}

(An operator $T$ is reductive if every subspace of $H$ that is invariant for $T$ is also invariant for $T^{*}$.)

Received by the editors May 1, 1980 and, in revised form, March 2, 1981.

1980 Mathematics Subject Classification. Primary 47B20; Secondary 47D25.

'During the preparation of this paper this author received partial support from the National Science Foundation (U.S.A.).

${ }^{2}$ During the preparation of this paper this author was visiting Indiana University and received financial support from the National Science Council (Republic of China). 
This was pointed out by Turner [23]. By Fuglede's Theorem, $T \in(\mathrm{dc})$ iff $\mathcal{Q}(T)=$ $W^{*}(T)$, the von Neumann algebra generated by $T$. It is a result due to Sarason [19] that this latter equation is equivalent to $T$ being reductive.

Also, [23] shows that every nonunitary isometry is in (dc).

It is a standard result that, for any operator $T, T=T_{0} \oplus T_{1}$, where $T_{0}$ is normal and $T_{1}$ has no reducing subspace on which it is normal. If the summand $T_{0}$ does not appear, that is, $T=T_{1}$, the operator $T$ is said to be pure. It will be shown that every pure quasinormal operator is in (dc) (Theorem 3.10). The proof of this theorem reveals a connection between pure quasinormal operators and nest algebras [5].

In $\$ 4$, a characterization of the arbitrary quasinormal operators in ( $\mathrm{dc}$ ) is given (Theorem 4.10). This characterization is obtained by an interesting analysis of the possible intertwining operators between a pure quasinormal operator and a normal operator. In particular, the question of whether the kernel of $1-T^{*} T$ is zero is shown to be relevant.

2. Some representation theorems for quasinormal operators. The characterization of quasinormal operators due to Arlen Brown [1] is well known. There are further representations of special quasinormal operators that have escaped general recognition and are rather useful. In this section, two such representation theorems are presented and these are applied to the study of quasinormal operators.

Brown's theorem is stated for convenient reference. Recall that an operator is pure if there is no reducing subspace on which it is normal.

2.1. TheOREM [1]. An operator $T$ on $H$ is a pure quasinormal operator iff $T$ is unitarily equivalent to $S \otimes A$, where $S$ is the unilateral shift of multiplicity one and $A$ is a positive operator with $\operatorname{ker} A=\{0\}$.

If $A$ acts on a Hilbert space $L$, then $S \otimes A$ acts on $L^{(\infty)} \equiv L \oplus L \oplus \cdots$, and the action of $S \otimes A$ on $L^{(\infty)}$ is given by the matrix

$$
S \otimes A=\left[\begin{array}{ccccc}
0 & & & & \\
A & 0 & & & \\
0 & A & 0 & & \\
0 & 0 & A & 0 & \ddots
\end{array}\right]
$$

If $A$ is reduced by the subspace $L_{1}$ of $L$, it is easy to see that $S \otimes A$ is reduced by $L_{1}^{(\infty)}$. So if $A=A_{1} \oplus A_{2} \oplus \cdots$, where each $A_{n}$ is cyclic, then $S \otimes A=\left(S \otimes A_{1}\right) \oplus$ $\left(S \otimes A_{2}\right) \oplus \cdots$. The first representation theorem that will be presented applies only to $S \otimes A$, where $A$ is cyclic. But first a characterization of these quasinormal operators is obtained.

2.3. Proposition. A pure quasinormal operator $T$ on $H$ is unitarily equivalent to $S \otimes A$, where $A$ is a cyclic positive operator, iff there is a vector $e$ in $H$ such that $H$ is the closed linear span of $\left\{|T|^{k} T^{j} e: k, j \geqslant 0\right\}$. (Recall that $|T|=\left(T^{*} T\right)^{1 / 2}$.) 
Proof. Assume that $H=L^{(\infty)}$ and $T=S \otimes A$ has the representation (2.2). If such a vector $e$ exists, let $e=\left(e_{0}, e_{1}, \ldots\right), e_{i}$ in $L$. Note that

$$
|T|=\left[\begin{array}{cccc}
A & 0 & & \\
0 & A & & \\
& & A & \\
& & & \ddots
\end{array}\right]
$$

and, for $j \geqslant 1$, the zeroth coordinate of $|T|^{k} T^{j} e$ is 0 . On the other hand, the zeroth coordinate of $|T|^{k} e$ is $A^{k} e_{0}$. Hence, $e_{0}$ is a cyclic vector for $A$.

Now suppose that $e_{0}$ is a cyclic vector for $A$ and put $e=\left(e_{0}, 0,0, \ldots\right)$ in $L^{(\infty)}$. It is easy to see that, for $k, j \geqslant 0$, every coordinate of $|T|^{k} T^{j} e$ is zero except the $j$ th one, and this coordinate is $A^{k+j} e_{0}=A^{k}\left(A^{j} e_{0}\right)$. Because $A$ has dense range $A^{j} e_{0}$ is a cyclic vector for $A$. Hence, $e$ satisfies the stated condition.

For a quasinormal operator $T$, let $R(T)$ be the norm closed algebra generated by $T$ and $|T|$; thus, $R(T)$ is an abelian algebra. Say that $T$ is $R(T)$-cyclic if there is a vector $e$ such that $\{R e: R \in R(T)\}$ is dense in $H$. By the preceding proposition, $T$ is $\Re(T)$-cyclic iff $A$ is cyclic.

The following notation will remain fixed throughout this paper. If $\nu$ is a positive Borel measure on $[0, \infty), \tilde{v}$ will denote the measure on $\mathbf{C}$ defined by

$$
d \tilde{\nu}\left(r e^{i \theta}\right)=\frac{1}{2 \pi} d \theta d \nu(r) .
$$

That is, $\tilde{\nu}$ is the measure such that, for every continuous nonnegative function $f$ on $\mathbf{C}$ with compact support,

$$
\int f(z) d \tilde{\nu}(x)=\frac{1}{2 \pi} \int_{0}^{\infty} \int_{0}^{2 \pi} f\left(r e^{i \theta}\right) d \theta d \nu(r) .
$$

2.4. THEOREM. If $T$ is a pure quasinormal operator that is $\Re(T)$-cyclic, then there is a measure $\nu$ on $[0,\|T\|]$ such that, if $\Re$ is the closed linear span of $\left\{|z|^{k} z^{j}: k, j \geqslant 0\right\}$ in $L^{2}(\tilde{\nu})$, then $T$ is unitarily equivalent to multiplication by $z$ on $R$.

Proof. To simplify the notation, assume that $\|T\|=1, H=L^{(\infty)}$, and $T=S \otimes A$. Moreover, since $A$ is cyclic, there is a positive measure $\nu$ on $[0,1]$ such that $A$ is unitarily equivalent to multiplication by the independent variable, $r$, on $L^{2}(\nu)$; assume $A=M_{r}$ and $L=L^{2}(\nu)$. Note that $\nu(\{0\})=0$.

For each $j \geqslant 0$, let $\Re_{j}$ be the closed linear span of $\left\{|z|^{k} z^{j}: k \geqslant 0\right\}$ in $L^{2}(\tilde{\nu})$. If $m \neq j$, then for $k, l \geqslant 0$,

$$
\left\langle|z|^{k} z^{j},|z|^{l} z^{m}\right\rangle_{L^{2}(\tilde{\nu})}=\left[\int_{0}^{1} r^{k+l+j+m} d \nu(r)\right]\left[\frac{1}{2 \pi} \int_{0}^{2 \pi} e^{i(j-m) \theta} d \theta\right]=0 .
$$

So $\Re_{j} \perp \Re_{m}$ for $j \neq m$. Clearly $\Re=\Re_{0} \oplus \Re_{1} \oplus \cdots$.

For $f$ in $L^{2}(\nu)$ and $j \geqslant 0$, define $g$ on $\mathbf{C} \backslash\{0\}$ by

$$
g(z)=|z|^{-j} z^{j} f(|z|) \text {. }
$$

Note that this defines $g$ a.e. [ $\tilde{\nu}]$ (because $\tilde{\nu}(\{0\})=0$ ). Also $\int|g|^{2} d \tilde{\nu}=\int|f|^{2} d \nu$. Thus, if $V_{j}: L^{2}(\nu) \rightarrow L^{2}(\tilde{\nu})$ is defined by

$$
\left(V_{j} f\right)(z)=|z|^{-j} z^{j} f(|z|)
$$


$V_{j}$ is an isometry. Let $p$ be a polynomial such that $p^{(n)}(0)=0$ for $0 \leqslant n \leqslant j-1$; that is, $p(r)=a_{j} r^{j}+a_{j+1} r^{j+1}+\cdots+a_{m} r^{m}$. It is easy to check that $V_{j} p \in \Re_{j}$. However, the Stone-Weierstrass Theorem implies that any continuous function on $[0,1]$ that vanishes at 0 can be uniformly approximated by such polynomials. Because $\nu(\{0\})=0$, it follows that these polynomials are dense in $L^{2}(\nu)$. Thus $V_{j}: L^{2}(\nu) \rightarrow$ $\Re_{j}$.

On the other hand, if $g \in \Re_{j}$, there is a sequence of polynomials $\left\{p_{n}\right\}$ such that $\int\left|p_{n}(|z|) z^{j}-g(z)\right|^{2} d \tilde{\nu}(z) \rightarrow 0$ as $n \rightarrow \infty$. But

$$
\int_{0}^{1}\left|r^{j} p_{n}(r)-r^{j} p_{m}(r)\right|^{2} d \nu(r)=\int\left|z^{j} p_{n}(|z|)-z^{j} p_{m}(|z|)\right|^{2} d \tilde{\nu}(z) .
$$

So $\left\{r^{j} p_{n}\right\}$ is a Cauchy sequence in $L^{2}(\nu)$; let $f$ be the limit of the sequence $\left\{r^{j} p_{n}\right\}$ in $L^{2}(\nu)$. But $V_{j}\left(r^{j} p_{n}\right)=z^{j} p_{n}$; since $V_{j}$ is continuous, $V_{j}(f)=g$. So $V_{j}$ is an isometry of $L^{2}(\nu)$ onto $\Re_{j}$.

Define $V: H \rightarrow \Re$ by $V=V_{0} \oplus V_{1} \oplus \ldots$ Thus, $V$ is an isometry of $H$ onto $R$. Let $M$ denote multiplication by $z$ on $\Re$. If $k, j \geqslant 0,|z|^{k} z^{j} \in \Re_{j}$ and $V^{-1}\left(|z|^{k} z^{j}\right)=r^{k+j}$ $\left(\in L^{2}(\nu)\right)$. Therefore $T V^{-1}\left(|z|^{k} z^{j}\right)=T\left(r^{k+j}\right)=M_{r}\left(r^{k+j}\right)=r^{k+j+1}$, an element of the $j+1$ coordinate of $H=\left[L^{2}(\nu)\right]^{(\infty)}$. Hence

$$
\begin{aligned}
V T V^{-1}\left(|z|^{k} z^{j}\right) & =V_{j+1}\left(r^{k+j+1}\right)=|z|^{-j-1} z^{j+1}|z|^{k+j+1} \\
& =|z|^{k} z^{j+1}=M\left(|z|^{k} z^{j}\right) .
\end{aligned}
$$

This implies that $V T V^{-1}=M$.

The following is an immediate consequence of the preceding representation theorem, though a direct proof is also possible.

2.5. CoRollary. If $T$ is a pure quasinormal operator, $\|T\|=1$, and $\operatorname{ker}\left(1-T^{*} T\right)$ $=\{0\}$, then $T^{n} \rightarrow 0(S O T)$.

Proof. Since $\left\|T^{n}\right\| \leqslant 1$ for all $n$ and, as was mentioned prior to the statement of Proposition 2.3, $T=T_{1} \oplus T_{2} \oplus \ldots$, where, for each $k \geqslant 1, T_{k}$ is $\mathscr{R}\left(T_{k}\right)$-cyclic, it can be assumed that $T$ is $R(T)$-cyclic. Thus, we may assume that $T=$ multiplication by $z$ on $\Re$, where $\Re$ is defined as in the preceding theorem.

If $\nu$ is as in 2.4, then the condition that $\operatorname{ker}\left(1-T^{*} T\right)=\{0\}$ is equivalent to the condition that $\tilde{\nu}(\partial \mathbf{D})=0$. Thus $|z|^{n} \rightarrow 0$ a.e. $[\tilde{\nu}]$ as $n \rightarrow \infty$. The conclusion is now immediate by the Lebesgue Dominated Convergence Theorem.

The next corollary is a special case of a more general result [16]. It is presented here in this form because this statement will be needed in the last section.

2.6. Corollary. If $T$ is a pure quasinormal operator and $\|T\|=1$, then $T^{* n} \rightarrow 0$ (SOT).

Proof. If $\operatorname{ker}\left(1-T^{*} T\right)=\{0\}$, then proceed as in the proof of the preceding corollary. If $\operatorname{ker}\left(1-T^{*} T\right) \neq\{0\}$, then $T=T_{0} \oplus T_{1}$ where $T_{1}$ is a pure isometry and $T_{0}$ is a pure quasinormal operator with $\operatorname{ker}\left(1-T_{0}{ }^{*} T_{0}\right)=\{0\}$. The conclusion for this case follows by applying the preceding case to $T_{0}$ and the well-known fact that $T_{1}^{* n} \rightarrow 0$ (SOT) for any pure isometry $T_{1}$. 
The next representation theorem only applies to pure quasinormal operators $T$ such that $T^{*} T$ is invertible; equivalently, it applies to pure quasinormal operators $T$ that are unitarily equivalent to $S \otimes A$, where $A$ is invertible. This representation was shown to us by Gerard Keough who derived a more general representation theorem for invertible operator-valued, weighted shifts (unpublished), analogous to that obtained for scalar-valued weighted shifts (see [21]). We begin with a lemma.

2.7. LemMa. Let $A$ be a positive invertible operator on a Hilbert space $L$ and define $H_{A}^{2}$ to be the collection of all formal power series

$$
F(z)=\sum_{n=0}^{\infty} z^{n} f_{n},
$$

where $\left\{f_{n}\right\} \subseteq L$ and $\sum_{n=0}^{\infty}\left\|A^{n} f_{n}\right\|^{2}<\infty$. If $G(z)=\sum z^{n} g_{n}$ is in $H_{A}^{2}$, and $\langle F, G\rangle$ is defined by

$$
\langle F, G\rangle=\sum_{n=0}^{\infty}\left\langle A^{n} f_{n}, A^{n} g_{n}\right\rangle_{L},
$$

then $H_{A}^{2}$ is a Hilbert space; also, if $\left\|A^{-1}\right\|^{-1}=r$, each $F$ in $H_{A}^{2}$ is an analytic function on $\{z \in \mathbf{C}:|z|<r\}$ with values in $L$.

Proof. The fact that $H_{A}^{2}$ is a Hilbert space follows from the fact that $A$ is invertible; the details are left to the reader. Let $F(z)=\sum z^{n} f_{n} \in H_{A}^{2}$. If $|z|<r$ and $m$ is fixed,

$$
\begin{aligned}
\sum_{n=0}^{m}|z|^{n}\left\|f_{n}\right\| & =\sum_{n=0}^{m}|z|^{n}\left\|A^{-n} A^{n} f_{n}\right\| \\
& \leqslant \sum_{n=0}^{m}(|z| / r)^{n}\left\|A^{n} f_{n}\right\| \leqslant\left[\sum_{n=0}^{m}(|z| / r)^{2 n}\right]^{1 / 2}\|F\| .
\end{aligned}
$$

It follows that the series defining $F$ converges absolutely and uniformly on every disk $\{z:|z| \leqslant s\}$ for $s<r$.

2.8. THEOREM. If $T$ is a pure quasinormal operator and $T^{*} T$ is invertible, then there is a positive invertible operator $A$ such that $T$ is unitarily equivalent to multiplication by $z$ on $H_{A}^{2}$.

Proof. Assume that $T=S \otimes A$ on $H=L^{(\infty)}$. As was mentioned above, the fact that $T^{*} T$ is invertible is equivalent to the fact that $A$ is invertible. Define $U$ : $H_{A}^{2} \rightarrow L^{(\infty)}$ by $U F=\left(A^{n} f_{n}\right)_{n=0}^{\infty}$ if $F(z)=\sum z^{n} f_{n}$. It is clear that $U$ is an isometry. Also, if $\left(h_{n}\right)_{n=0}^{\infty} \in L^{(\infty)}$, let $f_{n}=A^{-n} h_{n}$ for $n \geqslant 0$; it is immediate that $F=\sum z^{n} f_{n}$ is in $H_{A}^{2}$ and $U F=\left(h_{n}\right)$. Hence, $U$ is an isomorphism.

Let $M$ denote the operator defined on $H_{A}^{2}$ by multiplication by $z$. If $F \in H_{A}^{2}$,

$$
\begin{aligned}
U M(F) & =U\left(\sum_{n=0}^{\infty} z^{n+1} f_{n}\right)=\left(0, A f_{0}, A^{2} f_{1}, \ldots\right) \\
& =T\left(f_{0}, A f_{1}, A^{2} f_{2}, \ldots\right)=T U(F) .
\end{aligned}
$$

So $U M U^{-1}=T$. 
3. Every pure quasinormal operator is in (dc). Unless otherwise stated, it is assumed throughout this section that $T$ is a pure quasinormal operator, $T=S \otimes A$, and $T$ is represented by the matrix (2.2) acting on $L^{(\infty)}=L_{0} \oplus L_{1} \oplus \cdots$, where $L_{n}=L$ for all $n \geqslant 0$. Also $A$ has dense range and $\operatorname{ker} A=\{0\}$.

3.1. Lemma. If $C=\left[C_{i j}\right]_{i, j=0}^{\infty} \in\{T\}^{\prime}$, then

(a) $C_{i j}=0$ for $j>i$, and

(b) $A C_{i j}=C_{i+1, j+1} A$ for $i \geqslant j$.

Conversely, if $C=\left[C_{i j}\right]$ is a bounded operator on $L^{(\infty)}$ satisfying (a) and (b), $C \in\{T\}^{\prime}$.

This lemma is well known and its proof is an exercise in matrix multiplication.

3.2. Lemma. If $D=\left[D_{i j}\right] \in\{T\}^{\prime \prime}$, then

(a) $D_{i j}=0$ for $j>i$, and

(b) $D_{i j}=D_{i+1, j+1} \in\{A\}^{\prime \prime}$ for $i \geqslant j$.

Proof. Since $\{T\}^{\prime \prime} \subseteq\{T\}^{\prime}$, Lemma 3.1 implies that (a) holds and $A D_{i j}$ $=D_{i+1, j+1} A$ for $i \geqslant j$. Also, if $B \in\{A\}^{\prime}$, let $C=\operatorname{diagonal}(B, B, \ldots)$. By Lemma 3.1, $C \in\{T\}^{\prime}$; hence $D C=C D$. A computation of the $(i, j)$ entry of both sides of this last equation yields the equation $D_{i j} B=B D_{i j}$. Thus $D_{i j} \in\{A\}^{\prime \prime}$. Since $\{A\}^{\prime \prime} \subseteq$ $\{A\}^{\prime}$, it follows that $A\left(D_{i j}-D_{i+1, j+1}\right)=0$. But $\operatorname{ker} A=\{0\}$; therefore $D_{i j}=$ $D_{i+1, j+1}$.

In order to show that $T \in(\mathrm{dc})$, it will be shown that if $D=\left[D_{i j}\right] \in\{T\}^{\prime \prime}$, then $D_{i j}$ is a multiple of $A^{i-j}$ when $i \geqslant j$. To do this it is necessary to find examples of operators in $\{T\}^{\prime}$. Some obvious examples can be obtained by taking matrices $\left[C_{i j}\right]$ where $C_{i j}=C_{i+1, j+1} \in\{A\}^{\prime}$ for $i \geqslant j$; that is, by requiring $C$ to be constant along its diagonals and to have that constant operator in $\{A\}^{\prime}$.

If $T=S \otimes A$ and $A$ is invertible, condition (b) of Lemma 3.1 states that $C_{i+1, j+1}=A C_{i j} A^{-1}$. An induction argument shows that the diagonal terms satisfy $C_{i i}=A^{i} C_{00} A^{-i}$ for all $i \geqslant 1$. So it is possible to manufacture an element $C$ of $\{T\}^{\prime}$ with nonzero entries only on the main diagonal if an operator $C_{00}$ can be found such that $\left\{\left\|A^{i} C_{00} A^{-i}\right\|\right\}$ is uniformly bounded. It is precisely this that will be done in the next lemma, even when $A$ is not invertible. (There is a connection here with nest algebras. See [5] and [13] and their bibliographies.)

Suppose $A$ is cyclic. Then there is a probability measure $\nu$ on the interval $[0, a]$, $a=\|A\|$, such that $A \cong M_{r}$ on $L^{2}([0, a], \nu)$. Define an operator $V$ on $L^{2}(\nu)$ by

$$
V f(x)=\int_{[x, a]} f(r) d \nu(r)
$$

for each $f$ in $L^{2}(\nu)$. It is easy to see that $V$ is a bounded operator, and $\|V\| \leqslant 1$ (because $\nu$ is a probability measure). In fact, this is a consequence of Lemma 3.4 below, which shows much more.

Remember that $\operatorname{ker} A^{n}=\{0\}$, so $A^{n}$ has dense range and $A^{-n}$ is a densely defined linear transformation. 
3.4. LEMMA. If $A=M_{r}$ on $L^{2}([0, a], \nu)$ and $V$ is defined as in (3.3), then the densely defined transformation $A^{n} V A^{-n}$ extends to a bounded operator on $L^{2}(\nu)$ with $\left\|A^{n} V A^{-n}\right\| \leqslant 1$.

Proof. If $\mathscr{Q}=\left\{f \in L^{2}(\nu): f(t)=0\right.$ for $0 \leqslant t \leqslant b$, where $\left.0<b \leqslant a\right\}$, the $\mathscr{D}$ is a dense linear manifold in $L^{2}(\nu)$, since $\nu(\{0\})=0$. If $f \in \mathcal{D}, r^{-n} f \in L^{2}(\nu)$, so $A^{n} V A^{-n} f$ is well defined. Moreover, since $(r / t) \leqslant 1$ for $t$ in $[r, a]$,

$$
\begin{aligned}
\left\|A^{n} V A^{-n} f\right\|^{2} & =\int_{[0, a]}\left|r^{n} \int_{[r, a]} t^{-n} f(t) d \nu(t)\right|^{2} d \nu(r) \\
& \leqslant \int_{[0, a]}\left|\int_{[r, a]} f(t) d \nu(t)\right|^{2} d \nu(r) \\
& \leqslant \int_{[0, a]}\left(\int|f(t)|^{2} d \nu(t)\right)(\nu[r, a]) d \nu(r) \leqslant\|f\|^{2} .
\end{aligned}
$$

Because $\mathscr{D}$ is dense, $A^{n} V A^{-n}$ extends to a contraction.

The fact that the sequence $\left\{A^{n} V A^{-n}\right\}$ is uniformly bounded can be obtained by applying Corollary 2 of [17]. This weakened form of Lemma 3.4 will suffice for the application in the next lemma.

3.5. LEMMA. If $D \in\{T\}^{\prime \prime}$, then there are constants $\beta_{0}, \beta_{1}, \ldots$, such that, relative to the decomposition $L^{(\infty)}=L_{0} \oplus L_{1} \oplus \ldots, D$ has the representation

$$
D=\left[\begin{array}{ccc}
\beta_{0} & 0 & 0 \\
\beta_{1} A & \beta_{0} & 0 \\
\beta_{2} A^{2} & \beta_{1} A & \beta_{0} \\
\vdots & \vdots & \vdots
\end{array}\right] .
$$

Proof. By Lemma 3.2, $D=\left[D_{i j}\right]$ where $D_{i j}=0$ for $j>i$ and $D_{i j}=D_{i+1, j+1}$ for $j \geqslant i$. Put $D_{k}=D_{k 0}=D_{k+j, j}$ for $j \geqslant 0$ and $k \geqslant 0$.

By the spectral theorem, $A=A_{1} \oplus A_{2}$ on $L=M_{1} \oplus M_{2}$, where $A_{1}$ is cyclic; $A_{1} \cong M_{r}$ on $L^{2}(\nu)$, where $\nu$ is a scalar-valued spectral measure for $A$ [7]. By Lemma 3.4, there is an operator $V$ such that if $C_{n}=A_{1}^{n} V A_{1}^{-n} \oplus 0$ on $L=M_{1} \oplus M_{2}$ for $n \geqslant 0$, then $\left\|C_{n}\right\| \leqslant 1$ for all $n$. By Lemma 3.1,

$$
C=\left[\begin{array}{cccc}
C_{0} & & & \\
& C_{1} & & 0 \\
& & C_{2} & \\
& 0 & & \ddots
\end{array}\right]
$$

belongs to $\{T\}^{\prime}$. Hence $D C=C D$. A straightforward matrix computation using this last equation yields that $D_{n} C_{k}=C_{n+k} D_{n}$ for all $n, k \geqslant 0$. In particular, $D_{n} C_{0}=C_{n} D_{n}$ for all $n \geqslant 0$. 
Since $D_{n} \in\{A\}^{\prime \prime}$ by Lemma 3.2, there is a bounded Borel function $\phi_{n}$ on $[0,\|A\|]$ such that $D_{n}=\phi_{n}(A)$. Thus the equation $D_{n} C_{0}=C_{n} D_{n}$ becomes

$$
\phi_{n}\left(A_{1}\right) V=A_{1}^{n} V A_{1}^{-n} \phi_{n}\left(A_{1}\right) .
$$

Assume that $A_{1}=M_{r}$ on $L^{2}(\nu), a=\|A\|=\left\|A_{1}\right\|$. If $\mathscr{D}$ is defined as in the proof of Lemma 3.4 and $f \in \mathscr{D}$, then the preceding equation becomes

$$
\begin{aligned}
\phi_{n}(x) \int_{[x, a]} f(r) d \nu(r) & =x^{n} \int_{[x, a]} r^{-n} \phi_{n}(r) f(r) d \nu(r) \\
& =\int_{[x, a]}\left(\frac{x}{r}\right)^{n} \phi_{n}(r) f(r) d \nu(r),
\end{aligned}
$$

for almost all $x[\nu]$. A manipulation of this equation yields that

$$
\int_{[x, a]} f(r)\left[\phi_{n}(x)-\left(\frac{x}{r}\right)^{n} \phi_{n}(r)\right] d \nu(r)=0
$$

for almost all $x[\nu]$. If $\mathscr{D}_{0}$ is a countable dense subset of $\mathscr{D}$, then there is a Borel set $\Delta$ such that $\nu([0, a] \backslash \Delta)=0$ and (3.7) holds for $f$ in $\mathscr{D}_{0}$ and $x$ in $\Delta$. Hence, for $x$ in $\Delta$, $\phi_{n}(x) / x^{n}=\phi_{n}(r) / r^{n}$ for almost all $r \geqslant x[\nu]$. This implies that $\phi_{n}(x) x^{-n}$ is a constant function in $L^{\infty}(\nu)$; say $\phi_{n}(x) x^{-n}=\beta_{n}$. Hence $D_{n}=\phi_{n}(A)=\beta_{n} A^{n}$.

No originality is claimed for the next lemma, though a reference does not seem to be available.

3.8. LEMMA. If a bounded operator $D$ has the representation (3.6), then $\sum_{n=0}^{\infty} \beta_{n} z^{n}$ is the power series expansion of a bounded analytic function $\phi$ on the disk centered at $z=0$ having radius $\|A\|$ and $\|\phi\|_{\infty} \leqslant\|D\|$.

Proof. Using spectral theory, it is easy to see that it suffices to assume that $A=M_{r}$ on $L^{2}(\nu)$, where $\nu$ is a probability measure on $[0, a], a=\|A\|$. If $f \in L^{2}(\nu)$ and $\left(\alpha_{0}, \alpha_{1}, \ldots\right) \in l^{2}$, then $\hat{f}=\alpha_{0} f \oplus \alpha_{1} f \oplus \cdots \in L^{2}(\nu) \oplus L^{2}(\nu) \oplus \cdots$ Thus $\|D \hat{f}\|$ $\leqslant\|D\|\|\hat{f}\|=\|D\|\|f\|\left[\Sigma\left|\alpha_{j}\right|^{2}\right]^{1 / 2}$. Since $D$ has the form (3.6), a computation shows that the $n$th coordinate of the vector $D \hat{f}$ is given by $(D f)_{n}=\left(\sum_{k=0}^{n} \beta_{k} \alpha_{n-k} A^{k}\right) f$. Hence

$$
\begin{aligned}
\|D\|^{2}\|f\|^{2} \sum_{j=0}^{\infty}\left|\alpha_{j}\right|^{2} \geqslant\|D \hat{f}\|^{2}=\sum_{n=0}^{\infty}\left\|(D \hat{f})_{n}\right\|^{2} \\
=\sum_{n=0}^{\infty}\left\|\left(\sum_{k=0}^{n} \beta_{k} \alpha_{n-k} A^{k}\right) f\right\|^{2} \\
=\sum_{n=0}^{\infty} \int\left|\sum_{k=0}^{n} \beta_{k} \alpha_{n-k} r^{k}\right|^{2}|f(r)|^{2} d \nu(r) \\
=\int\left[\sum_{n=0}^{\infty}\left|\sum_{k=0}^{n} \beta_{k} \alpha_{n-k} r^{k}\right|^{2}|f(r)|^{2}\right] d \nu(r) .
\end{aligned}
$$


Now $|f|^{2}$ is an arbitrary positive function in $L^{1}(\nu)$. Hence the preceding inequality implies that

$$
\sum_{n=0}^{\infty}\left|\sum_{k=0}^{n} \beta_{k} \alpha_{n-k}(\cdot)^{k}\right|^{2} \in L^{\infty}(\nu)
$$

and its $\nu$-essential supremum is bounded by $\|D\|^{2} \Sigma_{j=0}^{\infty}\left|\alpha_{j}\right|^{2}$.

This can be interpreted in the following way. There is a countable dense subset $M$ of the Hardy space $H^{2}$ and a Borel set $\Delta$ such that $\nu([0, a] \backslash \Delta)=0$ and if $r \in \Delta$ and $\alpha(z)=\sum \alpha_{n} z^{n} \in M$, then

$$
\sum_{n=0}^{\infty}\left(\sum_{k=0}^{n} \beta_{k} \alpha_{n-k} r^{k}\right) z^{n} \in H^{2}
$$

and the $H^{2}$ norm of this analytic function is bounded by $\|D\|\|\alpha\|$. It is a classical result that $\phi_{r}(z)=\sum \beta_{n} r^{n} z^{n}$ belongs to $H^{\infty}(\mathbf{D})$ for $r$ in $\Delta$ and $\left|\phi_{r}(z)\right| \leqslant\|D\|$ for $|z|<1$. Since $a=\|A\|$ belongs to the support of $\nu$, it follows that $\phi(z)=\sum_{n=0}^{\infty} \beta_{n} z^{n}$ is bounded and analytic on $\{z:|z|<a\}$.

3.9. Lemma. If $\|T\|=a$ and $D_{a}=\{z \in \mathrm{C}:|z|<a\}$, then for every function $\phi$ in $H^{\infty}\left(D_{a}\right)$, there is a well-defined operator $\phi(T)$ such that $\|\phi(T)\| \leqslant\|\phi\|_{\infty}$ and

(a) $\phi \rightarrow \phi(T)$ is an algebra homomorphism of $H^{\infty}\left(D_{a}\right)$ into $\mathcal{Q}(T)$.

(b) $z(T)=T$ and $1(T)=1$.

(c) If $\left\{\phi_{n}\right\}$ is a bounded sequence in $H^{\infty}\left(D_{a}\right)$ and $\phi_{n}(z) \rightarrow \phi(z)$ for all $z$ in $D_{a}$, then $\phi_{n}(T) \rightarrow \phi(T)$ WOT.

(d) If $\left\{\phi_{n}\right\}$ is as in part (c) and, in addition, $\phi_{n}\left(a e^{i t}\right) \rightarrow \phi\left(a e^{i t}\right)$ a.e., then $\phi_{n}(T) \rightarrow$ $\phi(T) S O T$.

The definition of this functional calculus is, when $a=1$, the functional calculus developed by Sz.-Nagy and Foias [14, p. 114] for contractions; the changes for $a \neq 1$ are trivial. All the details can be found in [14].

It is worth pointing out here that $\|\phi(T)\|=\|\phi\|_{\infty}$, and this is shown in Theorem 3.10 below. Also $\mathscr{Q}(T)=\left\{\phi(T): \phi \in H^{\infty}\left(D_{a}\right)\right\}$. This also is shown in Theorem 3.10. Both of these facts can be found by using the results of [3].

Now for the principal result of this paper.

3.10. TheOREM. If $T$ is a pure quasinormal operator, then $T$ is in $(d c)$. Moreover, if $\|T\|=a$ and $D_{a}=\{z \in \mathbf{C}:|z|<a\}$, the map $\phi \rightarrow \phi(T)$ is an isometric isomorphism of $H^{\infty}\left(D_{a}\right)$ onto $\mathcal{Q}(T)=\{T\}^{\prime \prime}$.

Proof. For convenience, assume $\|T\|=1$.

By Lemma 3.9, $\left\{\phi(T): \phi \in H^{\infty}\right\} \subseteq \mathbb{Q}(T)$ and it is clear that $\mathscr{Q}(T) \subseteq\{T\}^{\prime \prime}$. Hence, to complete the proof of the theorem it suffices to show if $D \in\{T\}^{\prime \prime}$, $D=\phi(T)$ for some $\phi$ in $H^{\infty}$ and $\|D\| \geqslant\|\phi\|_{\infty}$. But Lemmas 3.5 and 3.8 imply that if $D \in\{T\}^{\prime \prime}, D$ has the form (3.6), where $\phi(z)=\Sigma \beta_{n} z^{n} \in H^{\infty}$ and $\|\phi\|_{\infty} \leqslant\|D\|$. So it remains to show that $D=\phi(T)$. This can be done by letting $f=$ $f_{0} \oplus f_{1} \oplus \cdots \oplus f_{m} \oplus 0 \oplus \cdots$ and $g=g_{0} \oplus g_{1} \oplus \cdots \oplus g_{m} \oplus 0 \oplus \cdots$ be elements of 
$L^{(\infty)}=L_{0} \oplus L_{1} \oplus \cdots$ and showing that $\langle[D-\phi(T)] f, g\rangle=0$. The details are left to the reader.

The identification of $\mathscr{Q}(T)$ and $H^{\infty}$ is well known and no originality is claimed for this. In fact, this identification could have been obtained by using the fact that quasinormal operators are subnormal and appealing to [3].

Lambert [11] and Lambert and Turner [12] have studied $Q(T),\{T\}^{\prime},\{T\}^{\prime \prime}$ and the double commutant property when $T$ is a weighted shift with invertible operator weights. In the case when $A$ is invertible, the proof that $T=S \otimes A$ is in (dc) could have been accomplished by using the results of [12] and our Lemma 3.4, together with some additional computations.

\section{Necessary and sufficient conditions for an arbitrary quasinormal operator to be} in (dc). There are normal operators that do not belong to (dc) (viz., the nonreductive ones), so it is not surprising that there are (impure) quasinormal operators that are not in $(\mathrm{dc})$. To give necessary and sufficient conditions for a quasinormal operator to belong to (dc), it is first necessary to study the commutant of an arbitrary quasinormal operator $Q$ by writing $Q=T \oplus N$, where $T$ is pure and $N$ is normal. This leads to a consideration of intertwining operators. If $T_{1}$ and $T_{2}$ are operators on $H_{1}$ and $H_{2}$, let $9\left(T_{1}, T_{2}\right)$ be the set of all operators $X: H_{1} \rightarrow H_{2}$ such that $X T_{1}=T_{2} X$.

The next result is the main tool for studying the double commutant property for the direct sum of two operators. It is due to Turner [23].

4.1. Lemma. Let $T_{1}$ and $T_{2}$ be operators on $H_{1}$ and $H_{2}$, and assume that

(a) $\left\{T_{1}\right\}^{\prime \prime}=Q\left(T_{1}\right)$.

(b) $\cup\left\{\operatorname{ran} X: X \in \mathscr{G}\left(T_{1}, T_{2}\right)\right\}$ is dense in $H_{2}$.

(c) If $S \in \mathbb{Q}\left(T_{1}\right)$, there is a sequence of polynomials $\left\{p_{n}\right\}$ such that $p_{n}\left(T_{1}\right) \rightarrow S$ in the strong operator topology and $\sup \left\{\left\|p_{n}\left(T_{2}\right)\right\|: n \geqslant 1\right\}<\infty$.

Then $\left\{T_{1} \oplus T_{2}\right\}^{\prime \prime}=Q\left(T_{1} \oplus T_{2}\right)$.

4.2. TheOREM. If $T=S \otimes A$ is a pure quasinormal operator with $\|T\|=1$ and $M$ is a cyclic operator on a Hilbert space $K$ with $\|M\|<1$, then there is an operator $X: L^{(\infty)} \rightarrow K$ such that $X T=M X$ and $\operatorname{ran} X$ is dense in $K$.

Proof. We first prove the special case where $A$ is invertible (or $T^{*} T$ is invertible) and $\|M\|<\left\|A^{-1}\right\|^{-1}$. Let $\|M\|<r<\left\|A^{-1}\right\|^{-1}$. For the proof of this case, we will use the representation of $T$ obtained in Theorem 2.8. So assume $T=$ multiplication by $z$ on $H_{A}^{2}$. By Lemma 2.7, if $F(z)=\sum z^{n} f_{n} \in H_{A}^{2}, F$ is a vector-valued analytic function on the disk $D_{r} \equiv\{z \in \mathbf{C}:|z|<r\}$. Fix $g$ in $L$ with $\|g\|=1$. Then

$$
F_{g}(z)=\langle F(z), g\rangle=\sum_{n=0}^{\infty}\left\langle f_{n}, g\right\rangle z^{n}
$$

is a scalar-valued analytic function on $D_{r}$. Since $\|M\|<r, \sum_{n=0}^{\infty}\left\langle f_{n}, g\right\rangle M^{n}=F_{g}(M)$ is a well-defined bounded operator. 
Let $e_{0}$ be a cyclic vector for $M$ with $\left\|e_{0}\right\|=1$, and define $X: H_{A}^{2} \rightarrow K$ by $X F=F_{g}(M) e_{0}$. Putting $\rho=\left\|A^{-1}\right\|\|M\|$, it follows that $\rho<1$. Hence

$$
\begin{aligned}
\|X F\| & =\left\|\sum_{n=0}^{\infty}\left\langle f_{n}, g\right\rangle M^{n} e_{0}\right\|=\left\|\sum_{n=0}^{\infty}\left\langle A^{n} f_{n}, A^{-n} g\right\rangle M^{n} e_{0}\right\| \\
& \leqslant \sum_{n=0}^{\infty}\left\|A^{n} f_{n}\right\| \rho^{n} \leqslant\left[\sum_{n=0}^{\infty}\left\|A^{n} f_{n}\right\|^{2}\right]^{1 / 2}\left[\sum_{n=0}^{\infty} \rho^{2 n}\right]^{1 / 2} \\
& =\left(1-\rho^{2}\right)^{-1 / 2}\|F\| .
\end{aligned}
$$

Therefore $X$ is bounded. Moreover

$$
\begin{aligned}
X T(F) & =X(z F)=X\left(\sum_{n=0}^{\infty} z^{n+1} f_{n}\right) \\
& =\sum_{n=0}^{\infty}\left\langle f_{n}, g\right\rangle M^{n+1} e_{0}=M X(F) .
\end{aligned}
$$

So $X \in \mathscr{T}(T, M)$. Also, by appropriately choosing $F, F_{g}$ can be made to equal any polynomial. Since $e_{0}$ is a cyclic vector, $\operatorname{ran} X$ is dense. This completes the proof of the first case.

For the general case, let $\|M\|<r<1$. Since $\|A\|=\|T\|=1$, the spectral theorem implies that $A=A_{1} \oplus A_{2}$ on $L=N_{1} \oplus N_{2}$, such that $\sigma\left(A_{1}\right) \subseteq[r, 1]$ and $\sigma\left(A_{2}\right) \subseteq[0, r]$. Thus $T=T_{1} \oplus T_{2}$ on $L^{(\infty)}=N_{1}^{(\infty)} \oplus N_{2}^{(\infty)}$, where $T_{j}=S \otimes A_{j}(j=$ 1,2). By the first part of this proof, there is an operator $X_{1}: N_{1}^{(\infty)} \rightarrow K$ such that $X_{1} T_{1}=M X_{1}$ and $\operatorname{ran} X_{1}$ is dense. If $X=X_{1} \oplus 0$, it is easy to check that $X$ has the desired properties.

The preceding result is related to one obtained by Sz.-Nagy and Foiaş [15]; they prove a similar theorem for $T=$ any unilateral shift, but they obtain an $X$ with ker $X=\{0\}$ as well as dense range.

The next lemma is known to many, but cannot be classified as a standard result. It is essentially a trick that has not been codified, though it is used repeatedly by people who work in weak* approximation.

4.3. Lemma. If $\phi \in H^{\infty}$, then there is a sequence of polynomials $\left\{p_{n}\right\}$ such that

(a) $\left\|p_{n}\right\|_{\infty} \leqslant\|\phi\|_{\infty}$ for all $n$.

(b) $p_{n}(z) \rightarrow \phi(z)$ as $n \rightarrow \infty$ for $|z|<1$.

(c) $p_{n}\left(e^{i t}\right) \rightarrow \phi\left(e^{i t}\right)$ a.e. as $n \rightarrow \infty$.

Proof. Let $q_{n}$ be the average of the first $n$ partial sums of the Taylor expansion of $\phi$. Then $\left\|q_{n}\right\|_{\infty} \leqslant\|\phi\|_{\infty}$ and $q_{n} \rightarrow \phi$ weak* in $L^{\infty}\left[10\right.$, p. 19]. Considering $q_{n}$ and $\phi$ as belonging to $H^{2}$, it follows that $q_{n} \rightarrow \phi$ weakly in $H^{2}$. Thus there is a sequence $\left\{p_{n}\right\}$ such that $p_{n}$ is a convex combination of $\left\{q_{1}, \ldots, q_{n}\right\}$ and $p_{n} \rightarrow \phi$ in the $H^{2}\left(L^{2}\right)$ norm. Because $p_{n}$ is a convex combination of $\left\{q_{1}, \ldots, q_{n}\right\},\left\|p_{n}\right\|_{\infty} \leqslant\|\phi\|_{\infty}$. Passing to a subsequence, if necessary, we obtain that $p_{n}\left(e^{i t}\right) \rightarrow \phi\left(e^{i t}\right)$ a.e. as $n \rightarrow \infty$. By integrating $p_{n}$ opposite the Poisson kernel, it follows that $p_{n}(z) \rightarrow \phi(z)$ as $n \rightarrow \infty$ when $|z|<1$. 
4.4. THEOREM. If $T$ is a pure quasinormal operator with $\|T\|=1$ and $M$ is a completely nonunitary normal contraction on $K$, then

$$
\{T \oplus M\}^{\prime \prime}=\mathbb{Q}(T \oplus M)=\left\{\phi(T) \oplus \phi(M): \phi \in H^{\infty}\right\} .
$$

Moreover, the map $\phi \rightarrow \phi(T) \oplus \phi(M)$ is an isometric isomorphism of $H^{\infty}$ onto $Q(T \oplus M)$.

Proof. Put $Q=T \oplus M$. If $\phi \in H^{\infty}$, then Theorem 3.10 implies that $\|\phi(T)\|=$ $\|\phi\|_{\infty}$. Also, $\|\phi(M)\| \leqslant\|\phi\|_{\infty}$ since $M$ is a normal contraction. Thus,

$$
\|\phi\|_{\infty}=\|\phi(T)\| \leqslant \max \{\|\phi(T)\|,\|\phi(M)\|\}=\|\phi(Q)\| \leqslant\|\phi\|_{\infty} .
$$

Hence $\phi \rightarrow \phi(Q)=\phi(T) \oplus \phi(M)$ is an isometry.

It will now be shown that the hypothesis of Lemma 4.1 is satisfied if $T_{1}=T$ and $T_{2}=M$. If $D \in \mathbb{Q}(T)$, then $D=\phi(T)$ for some $\phi$ in $H^{\infty}$. Let $\left\{p_{n}\right\}$ be a sequence of polynomials such that $\left\|p_{n}\right\|_{\infty} \leqslant\|\phi\|_{\infty}$ for all $n, p_{n}(z) \rightarrow \phi(z)$ as $n \rightarrow \infty$ for $|z|<1$, and $p_{n}\left(e^{i t}\right) \rightarrow \phi\left(e^{i t}\right)$ a.e. as $n \rightarrow \infty$ (Lemma 4.3). By Lemma 3.9, $p_{n}(T) \rightarrow \phi(T)=D$ (SOT). Since $M$ is a normal contraction, $\left\|p_{n}(M)\right\| \leqslant\|\phi\|_{\infty}$ for all $n$. So condition (c) of Lemma 4.1 is satisfied. Since (a) is satisfied by Theorem 3.10, it remains to verify condition (b).

Decompose $M$ as a direct sum of cyclic normal operators, $M=M_{1} \oplus M_{2} \oplus \cdots$, where $M_{n} \in \mathscr{B}\left(K_{n}\right)$. Let $M_{n}=\int z d E_{n}(z)$ be the spectral decomposition. If $0<r<1$, let $K_{n, r}=E_{n}(\{z:|z| \leqslant r\}) K_{n}$ and put $M_{n, r}=M_{n}\left|K_{n, r}=M\right| K_{n, r}$. So $M_{n, r}$ is a cyclic normal operator with $\left\|M_{n, r}\right\| \leqslant r<1$. By Theorem 4.2, there is an operator $X_{n, r}: L^{(\infty)} \rightarrow K_{n, r}$ such that $X_{n, r} T=M_{n, r} X_{n, r}$ and ran $X_{n, r}$ is dense in $K_{n, r}$. If $Y_{n, r}: L^{(\infty)} \rightarrow K=K_{1} \oplus K_{2} \oplus \cdots$ is defined by

$$
Y_{n, r}=X_{1, r} \oplus \cdots \oplus X_{n, r} \oplus 0 \oplus \cdots,
$$

then $Y_{n, r} \in \mathscr{S}(T, M)$. (Here $X_{n, r}$ is also used to denote the map of $L^{(\infty)}$ into $K_{n}$.) Because $M$ is completely nonunitary, $\cup\left\{\operatorname{ran} Y_{n, r}: n \geqslant 1,0<r<1\right\}$ is dense in $K$. Thus, (b) is satisfied.

According to Lemma 4.1, $Q=T \oplus M \in(\mathrm{dc})$. It remains to show that $Q(Q) \subseteq$ $\left\{\phi(Q): \phi \in H^{\infty}\right\}$. If $C \oplus D \in \mathbb{Q}(T \oplus M)$, then $C \in \mathbb{Q}(T)$. Hence, there is a $\phi$ in $H^{\infty}$ such that $C=\phi(T)$. Since $\phi(Q)=\phi(T) \oplus \phi(M)=C \oplus \phi(M) \in Q(Q), 0 \oplus$ $[D-\phi(M)] \in \mathbb{Q}(Q)$. So it must be shown that if $0 \oplus D \in \mathbb{Q}(Q), D=0$.

If $0 \oplus D \in \mathbb{Q}(Q)$, then there is a net of polynomials $\left\{p_{j}\right\}$ such that $p_{j}(Q)=p_{j}(T)$ $\oplus p_{j}(M) \rightarrow 0 \oplus D$ (SOT). If $X \in \mathscr{S}(T, M)$, then $X p_{j}(T)=p_{j}(M) X$ for all $j$. Taking limits it follows that $0=D X$. Since the ranges of the operators in $\mathscr{G}(T, M)$ have a dense linear span in $K, D=0$.

Let $M$ be any normal operator on a Hilbert space $K$ and let $M=\int z d E(z)$ be its spectral representation. Then $M$ can be decomposed as a direct sum

$$
M=M_{0} \oplus M_{a} \oplus M_{s} \oplus M_{1}
$$

on $K=K_{0} \oplus K_{a} \oplus K_{s} \oplus K_{1}$, where $M_{0}=M\left|E(\mathbf{D}) K, M_{1}=M\right| E\left(\mathbf{C} \backslash \mathbf{D}^{-}\right) K, M_{a} \oplus$ $M_{s}=M \mid E(\partial \mathbf{D}) K, M_{a}$ has a spectral measure absolutely continuous with respect to Lebesgue measure on $\partial \mathbf{D}$, and $M_{s}$ has a spectral measure that is singular to Lebesgue measure on $\partial \mathbf{D}$. Of course, some of these summands may not appear. 
4.6. Lemma. Let $M$ be a normal contraction and let $M=M_{0} \oplus M_{a} \oplus M_{s}$ be as in (4.5). If $T$ is a pure quasinormal operator with $\|T\|=1$, then

$$
\begin{aligned}
\mathscr{Q}(T \oplus M) & =\mathscr{Q}\left(T \oplus M_{0} \oplus M_{a}\right) \oplus \mathbb{Q}\left(M_{s}\right) \\
& =\left\{\phi\left(T \oplus M_{0} \oplus M_{a}\right): \phi \in H^{\infty}\right\} \oplus\left\{\psi\left(M_{s}\right): \psi \in L^{\infty}\left(\mu_{s}\right)\right\},
\end{aligned}
$$

where $\mu_{s}$ is a scalar-valued spectral measure for $M_{s}$.

Proof. If $\phi \in H^{\infty}$ and $\psi \in L^{\infty}\left(\mu_{s}\right)$, then Lemma 4.3 of [20] implies there is a sequence of polynomials $\left\{p_{n}\right\}$ such that $\left|p_{n}(z)\right| \leqslant\|\phi\|_{\infty}$ for $|z|<1,\left|p_{n}\left(e^{i \theta}\right)\right|$ $\leqslant\|\psi\|_{\infty}$ a.e. $\left[\mu_{s}\right], p_{n}(z) \rightarrow \phi(z)$ for $z$ in $\mathbf{D}$, and $p_{n} \rightarrow \psi$ weak* in $L^{\infty}\left(\mu_{s}\right)$. It follows that

$$
p_{n}(T \oplus M)=p_{n}\left(T \oplus M_{0} \oplus M_{a}\right) \oplus p_{n}\left(M_{s}\right) \rightarrow \phi\left(T \oplus M_{0} \oplus M_{a}\right) \oplus \psi\left(M_{s}\right) \text { (WOT), }
$$

so this latter operator belongs to $\mathbb{Q}(T \oplus M)$. Clearly, by Theorem 4.4, every operator in $\mathscr{Q}(T \oplus M)$ has this form.

Before stating the principal results, a few examples will serve to focus the reader's attention on the salient features of the theorems. It will help to keep these examples in mind when reading the results.

4.7. ExAmple. Let $S$ be the unilateral shift of multiplicity one, let $B$ be the bilateral shift of multiplicity one, and let $M$ be multiplication by $t$ on $L^{2}(2,3)$. If $Q=S \oplus B \oplus M$, then the fact that the polynomially convex hulls of $\sigma(S \oplus B)$ and $\sigma(M)$ are disjoint implies that $\mathbb{Q}(Q)=\mathbb{Q}(S \oplus B) \oplus \mathbb{Q}(M)[4]$. Hence

$$
\mathcal{Q}(Q)=\left\{\phi(S \oplus B): \phi \in H^{\infty}\right\} \oplus\left\{\psi(M): \psi \in L^{\infty}(2,3)\right\} .
$$

Also, it follows from Rosenblum's Theorem [18] that $\{Q\}^{\prime}=\{S \oplus B\}^{\prime} \oplus\{M\}^{\prime}$; thus $\{Q\}^{\prime \prime}=\{S \oplus B\}^{\prime \prime} \oplus\{M\}^{\prime \prime}=\{S \oplus B\}^{\prime \prime} \oplus W^{*}(M)$. Therefore $Q$ can be shown to be in (dc) if $S \oplus B$ can be shown to belong to (dc).

Now $X \in\{S \oplus B\}^{\prime}$ iff $X$ is a $2 \times 2$ matrix $\left[X_{i j}\right]$ such that $X_{11} \in\{S\}^{\prime}=\mathbb{Q}(S)$, $X_{22} \in\{B\}^{\prime}=W^{*}(B), X_{12} \in \mathscr{9}(B, S)$, and $X_{21} \in \mathscr{T}(S, B)$. Because $X_{12} B=S X_{12}$, $B^{* n} X_{12}^{*}=X_{12}^{*} S^{* n}$ for all $n \geqslant 1$. Hence, for $f$ in $H^{2}$, $\left\|X_{12}^{*} f\right\|=\left\|B^{* n} X_{12}^{*} f\right\|=$ $\left\|X_{12}^{*} S^{* n} f\right\| \leqslant\left\|X_{12}^{*}\right\|\left\|S^{* n} f\right\|$. But $S^{* n} \rightarrow 0$ (SOT); so $X_{12}=0$. It can be shown that

$$
\{S \oplus B\}^{\prime}=\left\{\left[\begin{array}{ll}
\phi(S) & 0 \\
M_{\theta} & \psi(B)
\end{array}\right]: \phi \in H^{\infty}, \psi \text { and } \theta \in L^{\infty}\right\},
$$

where $M_{\theta}: H^{2} \rightarrow L^{2}$ is defined by $M_{\theta} f=\theta f$. For the present it suffices to know that $\left[\begin{array}{ll}0 & 0 \\ 1 & 0\end{array}\right]$ belongs to $\{S \oplus B\}^{\prime}$.

If $X \in\{S \oplus B\}^{\prime \prime}$, then, because $1 \oplus 0$ and $0 \oplus 1 \in\{S \oplus B\}^{\prime}, X=\phi(S) \oplus \psi(B)$, where $\phi \in H^{\infty}$ and $\psi \in L^{\infty}$. But

$$
\begin{aligned}
0 & =\left[\begin{array}{cc}
\phi(S) & 0 \\
0 & \psi(B)
\end{array}\right]\left[\begin{array}{ll}
0 & 0 \\
1 & 0
\end{array}\right]-\left[\begin{array}{ll}
0 & 0 \\
1 & 0
\end{array}\right]\left[\begin{array}{cc}
\phi(S) & 0 \\
0 & \psi(B)
\end{array}\right], \\
0 & =\left[\begin{array}{cc}
0 & 0 \\
M_{\psi}-M_{\phi} & 0
\end{array}\right] .
\end{aligned}
$$

Hence $\psi=\phi$ and $\{S \oplus B\}^{\prime \prime}=\left\{\phi(S) \oplus \phi(B): \phi \in H^{\infty}\right\}=\mathscr{Q}(S \oplus B)$.

It follows that $Q \in(\mathrm{dc})$. 
4.8. Example. Let $A=$ multiplication by $t$ on $L^{2}(0,1), T=S \otimes A, B=$ the bilateral shift of multiplicity one, and $M=$ multiplication by $t$ on $L^{2}(2,3)$. If $Q=T \oplus B \oplus M$, then, by Theorem 2.1 of [4],

$$
\begin{aligned}
\mathscr{Q}(Q) & =\mathscr{Q}(T \oplus B) \oplus \mathbb{Q}(M) \\
& =\left\{\phi(T \oplus B): \phi \in H^{\infty}\right\} \oplus\left\{\psi(M): \psi \in L^{\infty}(2,3)\right\} .
\end{aligned}
$$

If $X \in \mathscr{G}(B, T)$, then $X B^{n}=T^{n} X$. Hence, $X^{*} T^{* n}=B^{* n} X^{*}$. So $\left\|X^{*} f\right\|$ $=\left\|X^{*} T^{* n} f\right\|$. But $T^{* n} \rightarrow 0$ (SOT) by Corollary 2.6, so $X=0$. If $Y \in \mathscr{G}(T, B)$, then $Y T^{n}=B^{n} Y$. But $\operatorname{ker}\left(1-T^{*} T\right)=\{0\}$, so $T^{n} \rightarrow 0$ (SOT) by Corollary 2.5. Hence $Y=0$. Therefore $\{T \oplus B\}^{\prime}=\{T\}^{\prime} \oplus\{B\}^{\prime}$ and $\{T \oplus B\}^{\prime \prime}=\{T\}^{\prime \prime} \oplus\{B\}^{\prime \prime}$. Hence

$$
\begin{aligned}
\{Q\}^{\prime \prime} & =\{T\}^{\prime \prime} \oplus\{B\}^{\prime \prime} \oplus\{M\}^{\prime \prime} \\
& =\left\{\phi(T): \phi \in H^{\infty}\right\} \oplus\left\{\psi(B): \psi \in L^{\infty}\right\} \oplus\left\{\theta(M): \theta \in L^{\infty}(2,3)\right\} .
\end{aligned}
$$

Therefore $Q \notin(\mathrm{dc})$.

Examples 4.7 and 4.8 show that if $Q=T \oplus M$, where $T$ is a pure quasinormal operator with $\|T\|=1$, and $M$ is a normal operator with the decomposition (4.5), and if $M_{a} \neq 0$, the answer to whether $Q \in(\mathrm{dc})$ will depend on the answer to whether $\operatorname{ker}\left(1-T^{*} T\right) \neq\{0\}$. See Theorem 4.10 below.

4.9. Example. If $T=$ the unilateral shift, $\Delta=\{z \in \mathbf{C}:|z-1|<1,|z|>1\}$, $\mu=$ arc length measure on $\partial \Delta \cap\{z:|z-1|=1\}$, and $M=$ multiplication by $z$ on $L^{2}(\mu)$, put $Q=T \oplus M$. If $G=\operatorname{int}\left(\mathbf{D}^{-} \cup \Delta^{-}\right)$, then it is not difficult to see [3] that

$$
\mathcal{Q}(Q)=\left\{\phi(T) \oplus \phi(M): \phi \in H^{\infty}(G)\right\} \text {. }
$$

If $\varepsilon>0$, let $\Delta_{\varepsilon}=\{z \in \Delta:|z|>1+\varepsilon\}$. By Rosenblum's Theorem [17], $9\left(T, M \mid L^{2}\left(\mu \mid \partial \Delta_{\varepsilon}\right)\right)=\{0\}=\mathscr{}\left(M \mid L^{2}\left(\mu \mid \partial \Delta_{\varepsilon}\right), T\right)$. Since $\varepsilon$ was arbitrary and $\mu(\partial \mathbf{D})=0,9(T, M)=\mathscr{G}(M, T)=\{0\}$. Hence $\{T \oplus M\}^{\prime}=\{T\}^{\prime} \oplus\{M\}^{\prime}$, so

$$
\{Q\}^{\prime \prime}=\{T\}^{\prime \prime} \oplus\{M\}^{\prime \prime}=\left\{\phi(T): \phi \in H^{\infty}\right\} \oplus\left\{\psi(M): \psi \in L^{\infty}(\mu)\right\} .
$$

Therefore $Q \notin(\mathrm{dc})$.

Note that in Example 4.9 $T$ and $M$ both belong to (dc), though $Q=T \oplus M$ does not. The difficulty here is that $Q(Q) \neq Q(T) \oplus \mathbb{Q}(M)$.

4.10. TheOREM. Let $T$ be a pure quasinormal operator with $\|T\|=1$ and let $M$ be a normal operator with $M=M_{0} \oplus M_{a} \oplus M_{2} \oplus M_{1}$ as in (4.5). Put $Q=T \oplus M$.

(a) If $\operatorname{ker}\left(1-T^{*} T\right)=\{0\}, Q \in(d c)$ iff:

$\left(\mathrm{a}_{1}\right) M_{a}=0$.

$\left(\mathrm{a}_{2}\right) \mathbb{Q}(Q)=\mathbb{Q}\left(T \oplus M_{0} \oplus M_{s}\right) \oplus \mathbb{Q}\left(M_{1}\right)$.

$\left(\mathrm{a}_{3}\right) M_{1} \in(d c)$.

In this case,

$$
\begin{aligned}
\mathcal{Q}(Q)= & \left\{\phi\left(T \oplus M_{0}\right): \phi \in H^{\infty}\right\} \oplus\left\{\psi\left(M_{s}\right): \psi \in L^{\infty}\left(\mu_{s}\right)\right\} \\
& \oplus\left\{\theta\left(M_{1}\right): \theta \in L^{\infty}\left(\mu_{1}\right)\right\},
\end{aligned}
$$

where $\mu_{s}$ and $\mu_{1}$ are scalar-valued spectral measures for $M_{s}$ and $M_{1}$.

(b) If $\operatorname{ker}\left(1-T^{*} T\right) \neq\{0\}, Q \in(d c)$ iff:

$\left(\mathrm{b}_{1}\right) \mathbb{Q}(Q)=\mathbb{Q}\left(T \oplus M_{0} \oplus M_{a} \oplus M_{s}\right) \oplus \mathbb{Q}\left(M_{1}\right)$.

$\left(\mathrm{b}_{2}\right) M_{1} \in(d c)$. 
In this case,

$$
\begin{aligned}
\mathcal{Q}(Q)= & \left\{\phi\left(T \oplus M_{0} \oplus M_{a}\right): \phi \in H^{\infty}\right\} \oplus\left\{\psi\left(M_{s}\right): \psi \in L^{\infty}\left(\mu_{s}\right)\right\} \\
& \oplus\left\{\theta\left(M_{1}\right): \theta \in L^{\infty}\left(\mu_{1}\right)\right\},
\end{aligned}
$$

where $\mu_{s}$ and $\mu_{1}$ are scalar-valued spectral measures for $M_{s}$ and $M_{1}$.

Before proving this theorem, some observations about the conditions are appropriate. If $B$ is the bilateral shift of multiplicity one, then $N=B \otimes A$ is the minimal normal extension of $T=S \otimes A$. By Theorem 2.1 of [3], conditions $\left(\mathrm{a}_{2}\right)$ and $\left(b_{1}\right)$ are equivalent to

$\left(\mathrm{a}_{2}^{\prime}\right) \mathbb{Q}(N \oplus M)=\mathbb{Q}\left(N \oplus M_{0} \oplus M_{s}\right) \oplus \mathbb{Q}\left(M_{1}\right)$,

$\left(\mathrm{b}_{1}^{\prime}\right) \mathbb{Q}(N \oplus M)=\mathbb{Q}\left(N \oplus M_{0} \oplus M_{a} \oplus M_{s}\right) \oplus \mathbb{Q}\left(M_{1}\right)$.

In [2], necessary and sufficient conditions are given for $\left(\mathrm{a}_{2}^{\prime}\right)$ and $\left(\mathrm{b}_{1}^{\prime}\right)$ in terms of the scalar-valued spectral measures.

Proof of Theorem 4.10. Let $R=T \oplus M_{0} \oplus M_{a} \oplus M_{s}$ and let $M_{1}=\int z d E_{1}(z)$. If $\varepsilon>0$, let $\Delta_{\varepsilon}=\{z:|z| \geqslant 1+\varepsilon\}, K_{\varepsilon}=E_{1}\left(\Delta_{\varepsilon}\right) K_{1}$, and $M_{\varepsilon}=M_{1} \mid K_{\varepsilon}$. Since $\sigma\left(M_{\varepsilon}\right) \cap$ $\mathbf{D}^{-}=\square, \mathscr{G}\left(R, M_{\varepsilon}\right)=\mathscr{G}\left(M_{\varepsilon}, R\right)=\{0\}$. If $X \in \mathscr{Q}\left(R, M_{1}\right)$, let $X_{\varepsilon}=E_{1}\left(\Delta_{\varepsilon}\right) X$; so $X_{\varepsilon} \in$

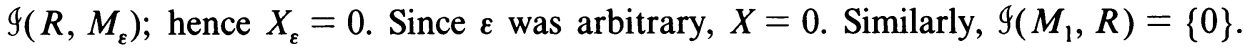
Hence, $\left\{R \oplus M_{1}\right\}^{\prime}=\{R\}^{\prime} \oplus\left\{M_{1}\right\}^{\prime}$. Using Lemma 4.6, it follows that

$$
\begin{aligned}
\{Q\}^{\prime \prime} & =\left\{T \oplus M_{0} \oplus M_{a}\right\}^{\prime \prime} \oplus\left\{M_{s}\right\}^{\prime \prime} \oplus\left\{M_{1}\right\}^{\prime \prime} \\
& =\left\{T \oplus M_{0} \oplus M_{a}\right\}^{\prime \prime} \oplus W^{*}\left(M_{s}\right) \oplus W^{*}\left(M_{1}\right) .
\end{aligned}
$$

(a) Assume $\operatorname{ker}\left(1-T^{*} T\right)=\{0\}$.

Suppose $\left(\mathrm{a}_{1}\right),\left(\mathrm{a}_{2}\right)$, and $\left(\mathrm{a}_{3}\right)$ hold. By $\left(\mathrm{a}_{2}\right)$ and Lemma 4.6, $\mathscr{Q}(\mathcal{Q})=\mathscr{Q}\left(T \oplus M_{0}\right) \oplus$ $W^{*}\left(M_{s}\right) \oplus \mathbb{Q}\left(M_{1}\right)$. By $\left(\mathrm{a}_{3}\right)$,

$$
\mathcal{Q}(Q)=\mathbb{Q}\left(T \oplus M_{0}\right) \oplus W^{*}\left(M_{s}\right) \oplus W^{*}\left(M_{1}\right) .
$$

Since $T \oplus M_{0} \in(\mathrm{dc})$ by Theorem 4.4, this last equation and (4.11) with $M_{a}=0$ imply $Q(Q)=\{Q\}^{\prime \prime}$.

Now suppose $Q \in(\mathrm{dc})$. From (4.11) and Lemma 4.6 it follows that

$$
\begin{aligned}
\left\{T \oplus M_{0} \oplus M_{a}\right\}^{\prime \prime} & \oplus W^{*}\left(M_{s}\right) \oplus\left\{M_{1}\right\}^{\prime \prime} \\
& =\mathscr{Q}(Q) \subseteq \mathbb{Q}\left(T \oplus M_{0} \oplus M_{a}\right) \oplus W^{*}\left(M_{s}\right) \oplus \mathbb{Q}\left(M_{1}\right) .
\end{aligned}
$$

It is immediate that $\left(\mathrm{a}_{3}\right)$ holds and

$$
\mathbb{Q}(Q)=\mathbb{Q}\left(T \oplus M_{0} \oplus M_{a}\right) \oplus W^{*}\left(M_{s}\right) \oplus \mathbb{Q}\left(M_{1}\right) \text {. }
$$

Moreover, $T \oplus M_{0} \oplus M_{a} \in(\mathrm{dc})$. But if $X \in \mathscr{Y}\left(T \oplus M_{0}, M_{a}\right)$, then $X\left(T \oplus M_{0}\right)^{n}=$ $M_{a}^{n} X$. But $T^{n} \oplus M_{0}^{n} \rightarrow 0$ (SOT) by Corollary 2.5 and the fact that $M_{0}$ is a completely nonunitary, normal contraction. So

$$
\|X f\|=\left\|M_{a}^{n} X f\right\|=\left\|X\left(T^{n} \oplus M_{0}^{n}\right) f\right\| \rightarrow 0 \quad \text { as } n \rightarrow \infty .
$$

Thus $X=0$. Similarly, $\mathscr{G}\left(M_{a}, T \oplus M_{0}\right)=\{0\}$. Hence $\left\{T \oplus M_{0} \oplus M_{a}\right\}^{\prime}=\left\{T \oplus M_{0}\right\}^{\prime}$ $\oplus\left\{M_{a}\right\}^{\prime}$ and $\left\{T \oplus M_{0} \oplus M_{a}\right\}^{\prime \prime}=\left\{T \oplus M_{0}\right\}^{\prime \prime} \oplus\left\{M_{a}\right\}^{\prime \prime}$. Since $T \oplus M_{0} \oplus M_{a} \in(\mathrm{dc})$, it must be that $\mathscr{Q}\left(T \oplus M_{0} \oplus M_{a}\right)=\mathscr{Q}\left(T \oplus M_{0}\right) \oplus \mathbb{Q}\left(M_{a}\right)$. But Lemma 4.6 implies that $\mathbb{Q}\left(T \oplus M_{0} \oplus M_{a}\right)$ is isomorphic to $H^{\infty}$, and, hence, cannot split. It must be that $M_{a}=0$. Since $\left(\mathrm{a}_{1}\right)$ holds, it is immediate from (4.12) that $\left(\mathrm{a}_{2}\right)$ is satisfied. 
(b) Assume that $\operatorname{ker}\left(1-T^{*} T\right) \neq\{0\}$.

Suppose that $\left(b_{1}\right)$ and $\left(b_{2}\right)$ are satisfied. Hence, combining Lemma 4.6 with these conditions, it follows that $\mathbb{Q}(Q)=\mathscr{Q}\left(T \oplus M_{0} \oplus M_{a}\right) \oplus W^{*}\left(M_{s}\right) \oplus W^{*}\left(M_{1}\right)$. Because of (4.11), it suffices to show that $T \oplus M_{0} \oplus M_{a} \in(\mathrm{dc})$. This will be done by applying Lemma 4.1 with $T_{1}=T \oplus M_{0}$ and $T_{2}=M_{a}$. Clearly condition (a) of 4.1 is satisfied; part (c) holds by using Lemma 4.6.

If $f \in \operatorname{ker}\left(1-T^{*} T\right)$, then, since $T\left(T^{*} T\right)=\left(T^{*} T\right) T, T^{*} T(T f)=T\left(T^{*} T\right) f=T f$. So $\operatorname{ker}\left(1-T^{*} T\right)$ is invariant for $T$. It is easy to see that the restriction of $T$ to this subspace must be an isometry. Since $\left(T^{*} T\right) T^{*}=T^{*}\left(T^{*} T\right)$, it follows that $\operatorname{ker}\left(1-T^{*} T\right)$ reduces $T$. Hence the restriction of $T$ to $\operatorname{ker}\left(1-T^{*} T\right)$ is a pure isometry. Therefore, $T=S \oplus R$ on $\mathcal{S} \oplus \Re$, where $S$ is a shift of multiplicity one. ( $\mathscr{R}$ contains part of $\operatorname{ker}\left(1-T^{*} T\right)$.)

Fix $e \neq 0$ in $K_{a}$ and put $N=$ the closed linear span of $\left\{M_{a}^{k} M_{a}^{* j} e: k, j \geqslant 0\right\}$. Thus, $M_{a} \mid N$ is unitarily equivalent to the operator $U$, multiplication by $z$ on $L^{2}(\eta)$, where $\eta(\cdot)=\left\langle E_{a}(\cdot) e, e\right\rangle$ and the unitary from $N$ onto $L^{2}(\eta)$ takes $e$ to 1. Assume $N=L^{2}(\eta)$ and $M_{a} \mid N=U$. Assume $\varsigma=H^{2}$ and $S$ is multiplication by $z$ on $H^{2}$. Define $X: H^{2} \oplus \Re \rightarrow L^{2}(\eta) \oplus N^{\perp}=K_{a}$ by $X(f \oplus g)=u v f \oplus 0$, where

(i) $u=(d \omega / d \eta)^{1 / 2}, \omega=$ Lebesgue measure on $\partial \mathbf{D}$;

(ii) $v \in L^{\infty}(\omega), v>0$ on $\partial \mathbf{D}$, and $\int \log v d \omega=-\infty$.

Thus, $\int|u v f|^{2} d \eta=\int|v|^{2}|f|^{2} d \omega \leqslant\|v\|_{\infty}^{2}\|f\|_{2}^{2}$. So $X$ is bounded. Also, $X\left(T \oplus M_{0}\right)=M_{a} X$. Finally, because $\int \log v d \omega=-\infty$, Szegö's Theorem [10] implies that $(\operatorname{ran} X)^{-}=L^{2}(\eta) \oplus\{0\}$. So condition (b) of Lemma 4.1 is satisfied. By that lemma, $T \oplus M_{0} \oplus M_{a} \in(\mathrm{dc})$.

Now suppose that $Q \in(\mathrm{dc})$. Thus, by (4.11),

$$
\begin{aligned}
&\left\{T \oplus M_{0} \oplus M_{a}\right\}^{\prime \prime} \oplus W^{*}\left(M_{s}\right) \oplus W^{*}\left(M_{1}\right)=\{Q\}^{\prime \prime}=\mathscr{Q}(Q) \\
& \subseteq \mathscr{Q}\left(T \oplus M_{0} \oplus M_{a}\right) \oplus \mathscr{Q}\left(M_{s}\right) \oplus \mathbb{Q}\left(M_{1}\right) \\
& \subseteq\left\{T \oplus M_{0} \oplus M_{a}\right\}^{\prime \prime} \oplus W^{*}\left(M_{s}\right) \oplus W^{*}\left(M_{1}\right) .
\end{aligned}
$$

Therefore, equality must hold in all these relations. Conditions $\left(b_{1}\right)$ and $\left(b_{2}\right)$ are now immediate.

4.13. COROLlaRY [23]. Every nonunitary isometry is in $(d c)$.

\section{BIBLIOGRAPHY}

1. A. Brown, On a class of operators, Proc. Amer. Math. Soc. 4 (1953), 723-728.

2. J. B. Conway, The direct sum of two normal operators, Indiana Univ. Math. J. 26 (1977), 277-289.

3. J. B. Conway and R. F. Olin, A functional calculus for subnormal operators. II, Mem. Amer. Math. Soc., No. 184 (1977).

4. J. B. Conway and $\mathrm{P}$. Y. Wu, The splitting of $Q\left(T_{1} \oplus T_{2}\right)$ and related questions, Indiana Univ. Math. J. 26 (1977), 41-56.

5. J. A. Deddens, Another description of nest algebras, Hilbert Space Operators, Lecture Notes in Math., vol. 693, Springer-Verlag, Berlin and New York, 1978, pp. 77-86.

6. J. A. Deddens and W. R. Wogen, On operators with the double commutant property, Duke Math. J. 43 (1976), 359-363.

7. R. G. Douglas, Banach algebra techniques in operator theory, Academic Press, New York, 1972.

8. N. Dunford and J. Schwartz, Linear operators. I, Interscience, New York, 1958.

9. A. Feintuch, Algebras generated by Volterra operators, J. Math. Anal. Appl. 56 (1976), 470-476.

10. K. Hoffman, Banach spaces of analytic functions, Prentice-Hall, Englewood Cliffs, N. J., 1962. 
11. A. L. Lambert, The algebra generated by an invertibly weighted shift, J. London Math. Soc. 5 (1972), $741-747$

12. A. L. Lambert and T. R. Turner, The double commutants of invertibly weighted shifts, Duke Math. J. 39 (1972), 385-389.

13. W. E. Longstaff, Generators of nest algebras, Canad. J. Math. 26 (1974), 565-575.

14. B. Sz.-Nagy and C. Foiaş, Harmonic analysis of operators on Hilbert space, North-Holland, Amsterdam, 1970.

15. _ Injection of shifts into strict contractions, Linear Operators and Approximation. II, Proc. M.R.I. Oberwolfach, Birkhäuser, Basel, 1974, pp. 29-37.

16. C. R. Putnam, Hyponormal contractions and strong power convergence, Pacific J. Math. 57 (1975), $531-538$.

17. M. Radjabalipour, Operators commuting with positive operators, Proc. Amer. Math. Soc. 77 (1979), $107-110$.

18. M. Rosenblum, On the operator equation $B X-X A=Q$, Duke Math. J. 23 (1956), 263-269.

19. D. Sarason, Invariant subspaces and unstarred operator algebras, Pacific J. Math. 17 (1966), 511-517. 20. , Weak-star density of polynomials, J. Reine Angew. Math. 252 (1972), 1-15.

21. A. L. Shields, Weighted shift operators and analytic function theory, Math. Surveys, vol. 13, Amer. Math. Soc., Providence, R. I., 1974, pp. 49-128.

22. T. R. Turner, Double commutants of algebraic operators, Proc. Amer. Math. Soc. 33 (1972), 415-419.

23. , Double commutants of isometries, Tôhoku Math. J. 24 (1972), 547-549.

24. M. Uchiyama, Double commutants of $C_{0}$ contractions, Proc. Amer. Math. Soc. 69 (1978), 283-288.

25. P. Y. Wu, Commutants of $C_{0}(N)$ contractions, Acta Sci. Math. (Szeged) 38 (1976), 193-202.

DEPARTMENT OF MATHEMATICS, Indiana University, BloOMington, INDiana 47405

Department of Applied Mathematics, National Chiao Tung University, Hsinchu, Taiwan, CHINA 